\title{
Update on the large-scale cosmic-ray anisotropy search at the highest energies by the Telescope Array Experiment
}

\section{Toshihiro Fujii ${ }^{a, b, *}$ on behalf of the Telescope Array Collaboration}

(a complete list of authors can be found at the end of the proceedings)

${ }^{a}$ Hakubi Center for Advanced Research, Kyoto University, Sakyo-ku, Kyoto, Japan

${ }^{b}$ Graduate School of Science, Kyoto University, Sakyo-ku, Kyoto, Japan

E-mail: fujii@cr.scphys.kyoto-u.ac.jp

\begin{abstract}
The study of large-scale anisotropy at the highest energies is essential for understanding the transition from cosmic rays of galactic origin to those of extra-galactic origin, along with the magnetic fields in the galaxy and those beyond. Motivated by a significant detection of the large-scale anisotropy above $8 \mathrm{EeV}$ by the Pierre Auger Observatory (Auger), we had previously reported, using 11 years of Telescope Array (TA) surface array data, a result compatible both with that of Auger, and with an isotropic source distribution [1]. In this contribution, we will show the preliminary updated results using 12 years TA SD data to search for the large-scale anisotropy at the highest energies.
\end{abstract}

$37^{\text {th }}$ International Cosmic Ray Conference (ICRC 2021)

July 12th-23rd, 2021

Online - Berlin, Germany

\footnotetext{
*Presenter
} 


\section{Introduction}

Over 100 years after the discovery of cosmic rays, there are still many open questions concerning the origin and nature of ultrahigh-energy cosmic rays (UHECRs) [2, 3]. With small deflections of UHECRs by galactic and intergalactic magnetic fields, $\sim 5^{\circ} Z(E / 50 \mathrm{EeV})^{-1}$, (where $Z$ is the charge of nuclei [4]), a small-intermediate scale anisotropy ${ }^{1}$ is predicted at the highest energies. On the other hand, the distance to sources and deflections by magnetic fields are increased at lower energies around $10 \mathrm{EeV}$, but still allowing for the possibility of a large-scale anisotropy, such as one that might be approximated as a dipole [5-7].

\subsection{Telescope Array experiment}

The Telescope Array experiment (TA) is the largest cosmic-ray detector in the Northern hemisphere, located near the city of Delta, Utah, USA $\left(39.30^{\circ}\right.$ North and $112.91^{\circ}$ West, and at $\sim 1400 \mathrm{~m}$ above sea level) [8]. The surface detector array (SD) consists of 507 plastic scintillators of $3 \mathrm{~m}^{2}$ area deployed in a square grid with a $1.2 \mathrm{~km}$ spacing. The total acceptance is about $700 \mathrm{~km}^{2}$. Additional surface detectors, designed to provide a fourfold increase in observation area, collectively referred to a TA $\times 4$, have begun operation [9]. Fluorescence detector (FD) view the air space above the SD. The FD measures directly the calorimetric energy of an air shower from the energy deposited by particles in the shower during its longitudinal development [10].

The arrival direction of an UHECR is inferred as measured by the TA SD is evaluated from the relative difference in arrival time of the shower front at each surface detector (which are timesynchronized using GPS modules). The energy estimator of the TA SD is the particle density measured at a distance of $800 \mathrm{~m}$ from the air shower axis, called $S_{800}$. The $S_{800}$ parameter is converted to the primary energy as a function of zenith angle based on a Monte Carlo simulation using the CORSIKA software package [11]. The obtained energy is calibrated to the calorimetric energy measured by the fluorescence detectors using a scaling factor of 1/1.27 [12]. The typical resolution of the TA SD is $1.0^{\circ} \sim 1.5^{\circ}$ in arrival direction and $10 \% \sim 15 \%$ in primary energy [12], and the systematic uncertainty in the energy scale is quoted at $21 \%$ [13].

\subsection{Large-scale anisotropy search}

Recently, the Pierre Auger Collaboration reported the observation of a significant large-scale anisotropy in the arrival directions of cosmic rays above $8 \mathrm{EeV}$, indicating an obvious dipole structure of $4.7 \%$ amplitude in a projection of the right ascension with a 5.2 $\sigma$ significance [14]. An enhancement of the dipole amplitude above $4 \mathrm{EeV}$ and results down to $0.03 \mathrm{EeV}$ are also reported $[15,16]$. These results are consistent with an extragalactic origin of UHECRs. In the Northen hemisphere, the Telescope Array Collaboration reported a result consistent both with an isotropic source distribution, and with the dipole structure reported by Auger using 11 years TA SD data[1].

In this proceedings, we report the large-scale cosmic-ray anisotropy search at the highest energies and its energy dependences using 12 years TA SD data.

\footnotetext{
${ }^{1}$ In this research field, we define a small angular scale anisotropy as less than $5^{\circ}$, intermediate scale as $10^{\circ}$ to $35^{\circ}$ and large scale as $>40^{\circ}$.
} 


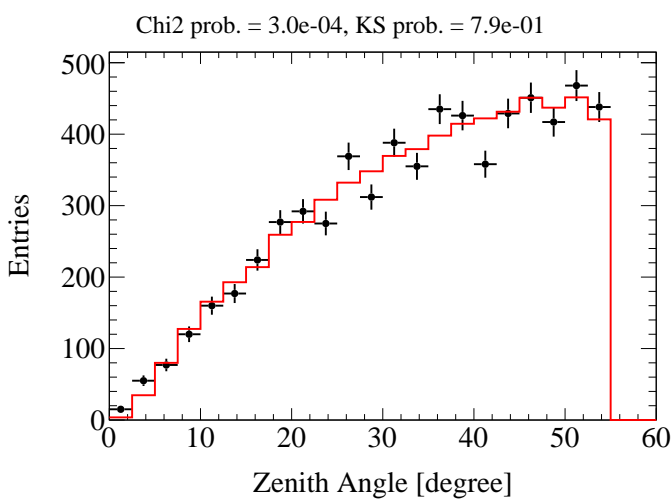

(a) Zenith angle

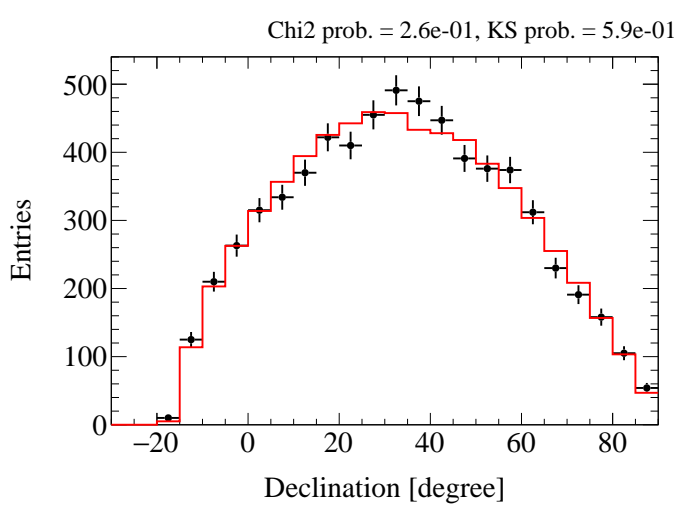

(c) Declination

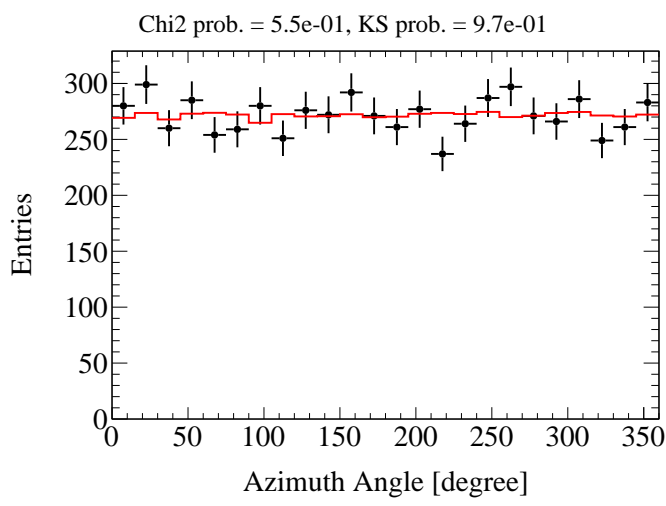

(b) Azimuth angle

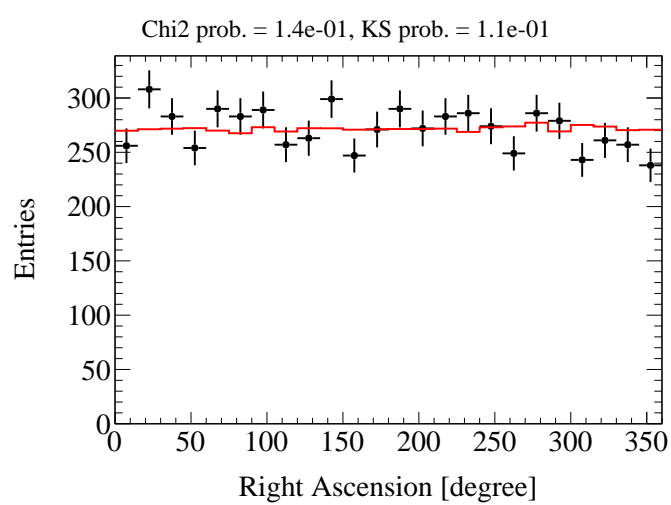

(d) Right Ascension

Figure 1: Parameter comparisons between the measured distribution and expectation evaluated from the MC simulations. Probabilities of the statistical tests using $\chi^{2}$ test and Kolmogorov-Smirnov test are described on the top of the figures.

\section{Data-set and methodology}

The TA SD data recorded over 12 years from May 2008 to May 2020 were used for a study of the large-scale anisotropy. To apply the same cut with our earlier publication, we use an a priori energy threshold of $8.8 \mathrm{EeV}$, equivalent to $8 \mathrm{EeV}$ used by Auger, accounting for $10 \%$ energy scale difference between the two experiments [17].

There were 6518 events above $8.8 \mathrm{EeV}$, with zenith angles less than $55^{\circ}$ and passing the same quality cuts used in the TA spectrum analysis [12]. In this data-set, TA SD is capable of measuring UHECRs were injected in a declination band from $-15^{\circ}$ to $90^{\circ}$. As the trigger efficiency of the TA SD below $10 \mathrm{EeV}$ is less than $100 \%$, the obtained right ascension distribution is compared with the expected distribution produced an isotropic UHECR sky, and from a time-dependent Monte Carlo (MC) simulation including actual calibration constants, live time and dead time of each surface detector station, and TA SD trigger efficiencies.

Before investigating large-scale cosmic-ray anisotropy, consistencies of the parameter distribu- 


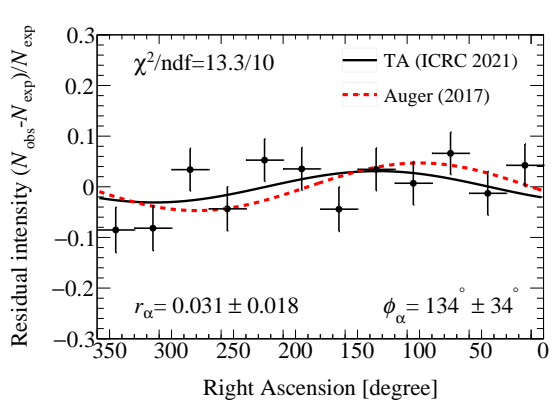

(a) Equatorial dipole

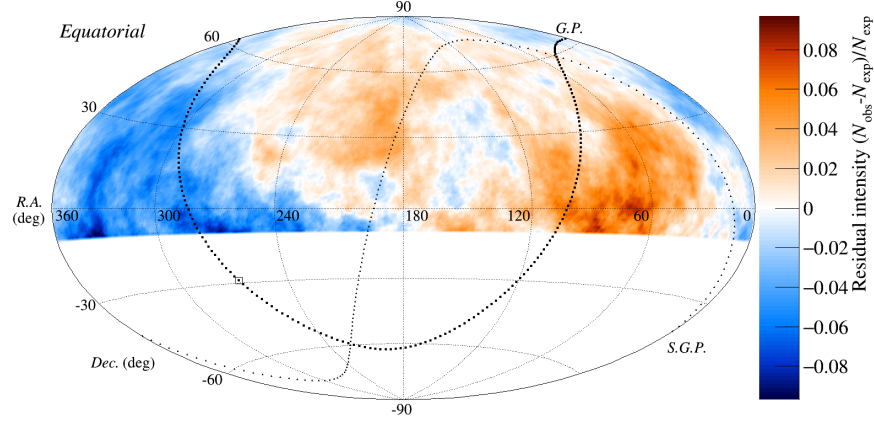

(b) Residual-intensity sky-map

Figure 2: (a) Residual intensities of UHECRs with energies above $8.8 \mathrm{EeV}$ observed with 12 years of TA data as a function of the right ascension. The black curve shows the TA fitted dipole result and and the red dashed curve represents the dipole reported by Auger. (b) Sky map of residual intensities between TA data and an isotropic distribution shown in the Equatorial and the Galactic coordinates. The arrival directions are oversampled with a $45^{\circ}$ radius cylindrical function. The galactic plane (G.P.) and the super-galactic plane (S.G.P.) are shown as thick and thin dotted curves, respectively. The galactic center (G.C.) is indicated by the open square.

tions between observed data and MC simulation are first verified. Figure 1 shows distributions of zenith angle, azimuth angle, declination and right ascension for the 12 years TA SD data compared with expectations evaluated from the time-dependent MC simulation. In all four cases the data and MC are in good agreement.

\section{Result}

Figure 2(a) shows the residual intensity as a function of right ascension between 12 years of TA SD data and the isotropic expectation calculated from the time-dependent MC simulation. The residuals are fitted to the form $r_{\alpha} \cos \left(x-\phi_{\alpha}\right)$, where $r_{\alpha}$ is the amplitude of the dipole and $\phi_{\alpha}$ is the phase. The obtained dipole structure has an amplitude $r_{\alpha}$ of $3.1 \pm 1.8 \%$ and a phase $\phi_{\alpha}$ of $134^{\circ} \pm 34^{\circ}$. The TA SD data points are also compared with (a) an isotropic distribution and (b) the dipole structure reported by Auger. The latter has a 4.7\% amplitude and a phase of $100^{\circ}$. With current statistics, the obtained TA SD result is consistent with an isotropic distribution. Since there is no significant dipole structure, an upper limit is evaluated for a chance probability of $1 \%$ that a fluctuation of an isotropic source distribution would yield an amplitude greater than the reference limit. The obtained $99 \%$ confidence-level upper-limit on amplitude is $7.6 \%$ for TA data above 8.8 EeV.

Figure 2(b) shows the sky map of residual intensities with oversampling of $45^{\circ}$ top-hat function to discern the structure of the dipole components. Although no significant amplitude is found, the direction of the limited dipole enhancement is far away from the Galactic center. This result would be more indicative of an extragalactic source distribution than a galactic one. Furthermore, we search for an energy dependence of the dipole components in four energy ranges above $4 \mathrm{EeV}$ as shown in Figure 3. There is no significant component of dipole structure in all energy ranges. 


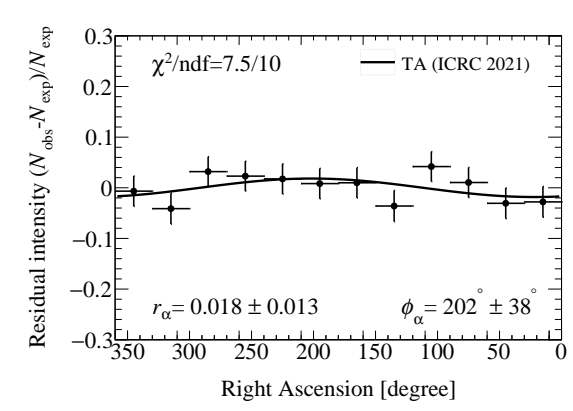

(a) $4 \mathrm{EeV} \leq E<8 \mathrm{EeV}$

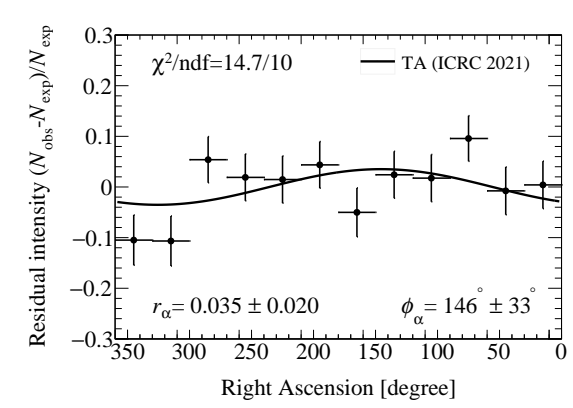

(c) $8 \mathrm{EeV} \leq E<16 \mathrm{EeV}$

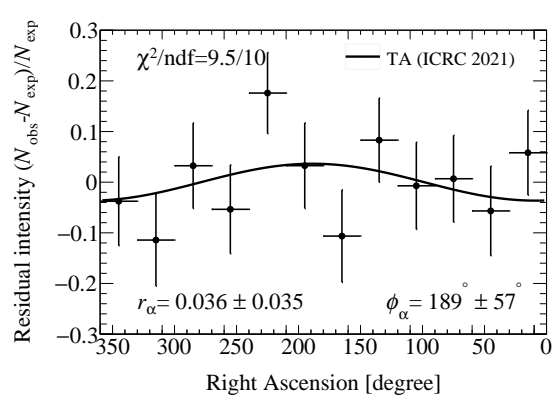

(e) $16 \mathrm{EeV} \leq E<32 \mathrm{EeV}$

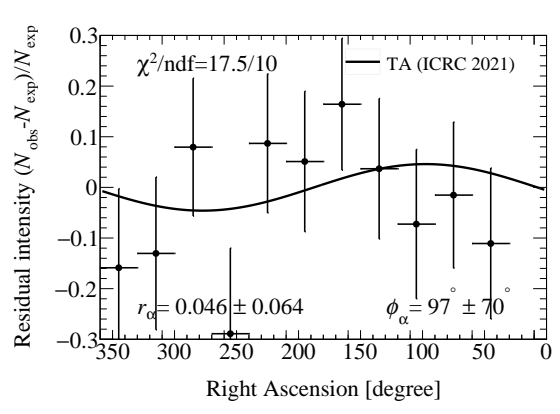

(g) $32 \mathrm{EeV} \leq E$

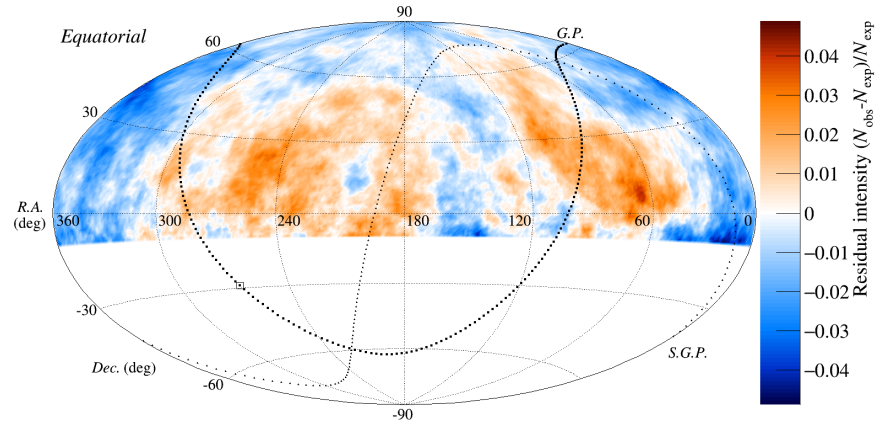

(b) $4 \mathrm{EeV} \leq E<8 \mathrm{EeV}$

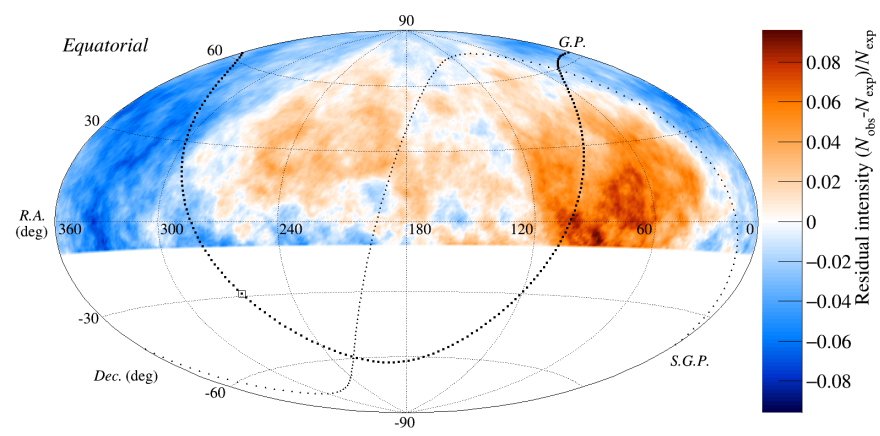

(d) $8 \mathrm{EeV} \leq E<16 \mathrm{EeV}$

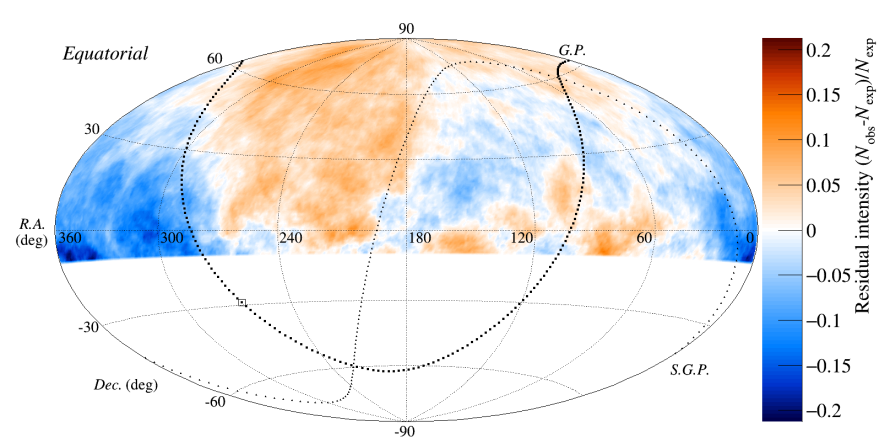

(f) $16 \mathrm{EeV} \leq E<32 \mathrm{EeV}$

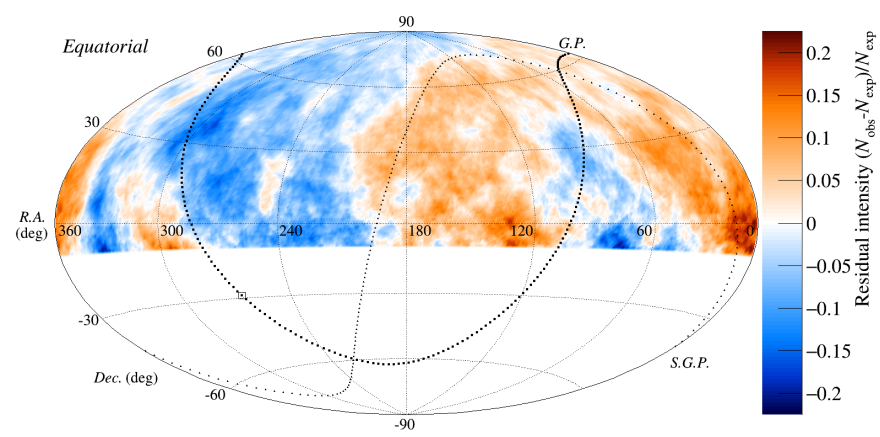

(h) $32 \mathrm{EeV} \leq E$

Figure 3: Preliminary results from 12 years of TA SD data of residual intensities vs right ascension (left) and skymap (right) in four energy ranges; (a,b) $4 \mathrm{EeV} \leq E<8 \mathrm{EeV}$, (c,d) $8 \mathrm{EeV} \leq E<16 \mathrm{EeV}$, (e,f) $16 \mathrm{EeV}$ $\leq E<32 \mathrm{EeV}$ and $(\mathrm{g}, \mathrm{h}) 32 \mathrm{EeV} \leq E$. 

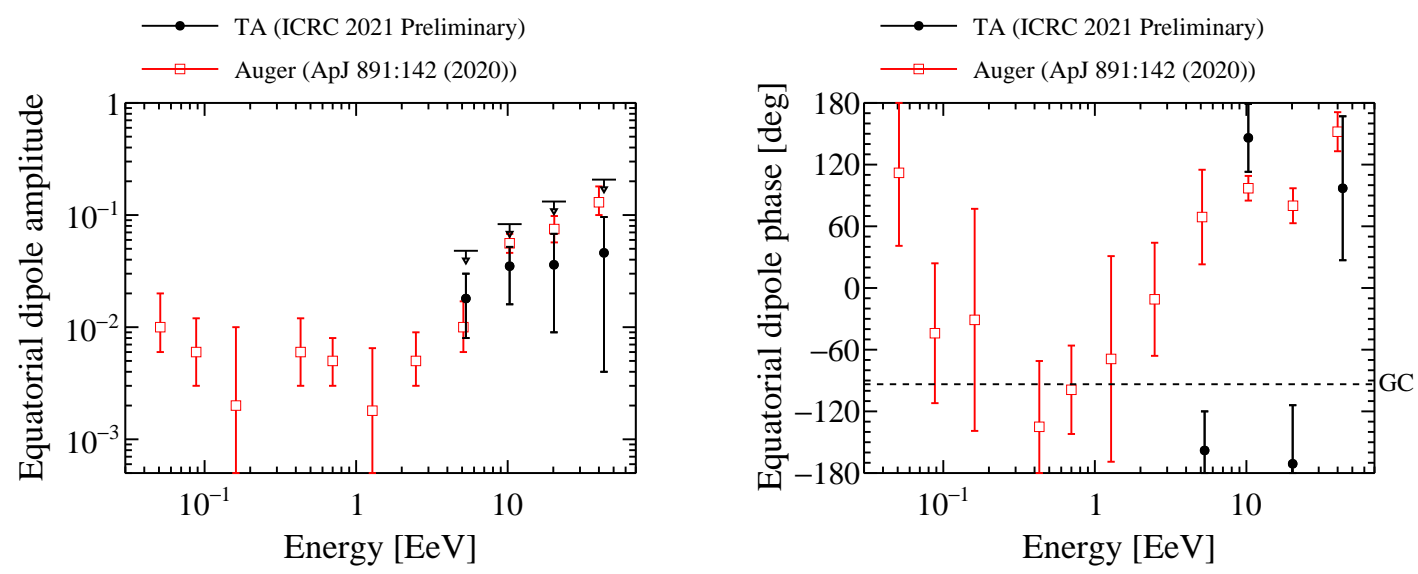

Figure 4: Preliminary result of equatorial dipole amplitude and phase measured with Telescope Array experiment from 12 years of data (black filled circles), compared to Auger reported result (red open squares) [16]. The phase toward the Galactic center (GC) is indicated as a dashed line.

Figure 4 shows the obtained preliminary result of amplitude and phase using 12 years TA SD data compared to the Auger reported result [16].

None of the measured TA data amplitudes are significantly different from zero, but their best-fit values appear to increase with energy, while the corresponding phases are all far from the Galactic center. Table 1 is the summary of preliminary results and their corresponding upper limit on amplitude with $99 \%$ confidence level.

\begin{tabular}{l|ccccc}
$E[\mathrm{EeV}]$ & $E_{\mathrm{med}}[\mathrm{EeV}]$ & $N$ & $r_{\alpha}[\%]$ & $\phi_{\alpha}\left[^{\circ}\right]$ & $r_{\alpha}^{\mathrm{UL}}[\%]$ \\
\hline \hline $8.8 \leq E$ & 13.0 & 6518 & $3.1_{-1.6}^{+1.6}$ & $134 \pm 34$ & 7.6 \\
\hline $4 \leq E<8$ & 5.3 & 13361 & $1.8_{-1.0}^{+1.2}$ & $202 \pm 38$ & 4.8 \\
$8 \leq E<16$ & 10.3 & 5446 & $3.5_{-1.9}^{+1.7}$ & $146 \pm 33$ & 8.3 \\
$16 \leq E<32$ & 20.2 & 1623 & $3.6_{-2}^{+3.2}$ & $189 \pm 57$ & 13.2 \\
$32 \leq E$ & 43.2 & 595 & $4.6_{-4.2}^{+5.0}$ & $97 \pm 70$ & 20.7
\end{tabular}

Table 1: Summary of preliminary dipole search result of 12 years TA SD data. The energy range, median energy, number of events, best-fit dipole amplitudes and phase angles, and $99 \%$ confidence upper limits of $r_{\alpha}^{\mathrm{UL}}$ are tabulated.

\section{Conclusion}

We report the results of the search for dipole structure in the arrival direction of UHECR using 12 years of TA SD data from the Northern sky and for possible energy dependence of the amplitudes. There are no significant large-scale anisotropies with the current limited statistics. We have evaluated a 99\% confidence-level upper limit of $r_{\alpha}^{\mathrm{UL}}=7.6 \%$ above $8.8 \mathrm{EeV}$ on the amplitude of a dipole structure in a projection of the right ascension. Although the tendency of amplitude and phase of the dipole structure shows a similar one reported from Auger, much greater statistics from 
TA are required to distinguish the two hypotheses. Future data collection by TA and the on-going upgrade of TA $\times 4$ will be essential for further studies.

\section{References}

[1] Telescope Array Collaboration, R. U. Abbasi et al., Search for Large-scale Anisotropy on Arrival Directions of Ultra-high-energy Cosmic Rays Observed with the Telescope Array Experiment, Astrophys. J. Lett. 898 (2020), no. 2 L28, [2007. 00023].

[2] B. R. Dawson, M. Fukushima, and P. Sokolsky, Past, Present and Future of UHECR Observations, PTEP 2017 (2017), no. 12 12A101, [1703.07897].

[3] R. Alves Batista et al., Open Questions in Cosmic-Ray Research at Ultrahigh Energies, Front. Astron. Space Sci. 6 (2019) 23, [1903.06714].

[4] J. Bray and A. Scaife, An upper limit on the strength of the extragalactic magnetic field from ultra-high-energy cosmic-ray anisotropy, Astrophys. J. 861 (2018), no. 1 3, [1805. 07995].

[5] D. Harari, S. Mollerach, and E. Roulet, Anisotropies of ultrahigh energy cosmic ray nuclei diffusing from extragalactic sources, Phys. Rev. D 92 (2015), no. 6 063014, [1507.06585].

[6] A. di Matteo and P. Tinyakov, How isotropic can the UHECR flux be?, Mon. Not. Roy. Astron. Soc. 476 (2018), no. 1 715-723, [1706. 02534].

[7] B. Eichmann and T. Winchen, Galactic Magnetic Field Bias on Inferences from UHECR Data, JCAP 04 (2020) 047, [2001. 01530].

[8] Telescope Array Collaboration, T. Abu-Zayyad et al., The surface detector array of the Telescope Array experiment, Nucl. Instrum. Meth. A689 (2013) 87-97, [1201.4964].

[9] Telsecope Array Collaboration, E. Kido et al., Status and prospects of the TAx4 experiment, EPJ Web Conf. 210 (2019) 06001.

[10] H. Tokuno et al., New air fluorescence detectors employed in the Telescope Array experiment, Nucl. Instrum. Meth. A676 (2012) 54-65, [1201.0002].

[11] D. Heck, J. Knapp, J. N. Capdevielle, G. Schatz, and T. Thouw, CORSIKA: A Monte Carlo code to simulate extensive air showers, Forschungszentrum Karlsruhe Report FZKA-6019 (1998).

[12] Telescope Array Collaboration, T. Abu-Zayyad et al., The Cosmic Ray Energy Spectrum Observed with the Surface Detector of the Telescope Array Experiment, Astrophys. J. 768 (2013) L1, [1205 . 5067].

[13] Telescope Array Collaboration, R. U. Abbasi et al., The energy spectrum of cosmic rays above $10^{17.2} \mathrm{eV}$ measured by the fluorescence detectors of the Telescope Array experiment in seven years, Astropart. Phys. 80 (2016) 131-140, [1511.07510]. 
[14] Pierre Auger Collaboration, A. Aab et al., Observation of a Large-scale Anisotropy in the Arrival Directions of Cosmic Rays above $8 \times 10^{18} \mathrm{eV}$, Science 357 (2017), no. 6537 1266-1270, [1709.07321].

[15] Pierre Auger Collaboration, A. Aab et al., Large-scale cosmic-ray anisotropies above 4 EeV measured by the Pierre Auger Observatory, Astrophys. J. 868 (2018), no. 1 4, [1808. 03579].

[16] Pierre Auger Collaboration, A. Aab et al., Cosmic-ray anisotropies in right ascension measured by the Pierre Auger Observatory, Astrophys. J. 891 (2020) 142, [2002 . 06172].

[17] V. Verzi, D. Ivanov, and Y. Tsunesada, Measurement of Energy Spectrum of Ultra-High Energy Cosmic Rays, PTEP 2017 (2017), no. 12 12A103, [1705 . 09111]. 


\section{Full Authors List: The Telescope Array Collaboration}

R.U. Abbasi ${ }^{1,2}$, T. Abu-Zayyad ${ }^{1,2}$, M. Allen ${ }^{2}$, Y. Arai ${ }^{3}$, R. Arimura ${ }^{3}$, E. Barcikowski ${ }^{2}$, J.W. Belz ${ }^{2}$, D.R. Bergman ${ }^{2}$, S.A. Blake ${ }^{2}$, I. Buckland ${ }^{2}$, R. $\mathrm{Cady}^{2}$, B.G. Cheon ${ }^{4}$, J. Chiba ${ }^{5}$, M. Chikawa ${ }^{6}$, T. Fujii ${ }^{7}$, K. Fujisue ${ }^{6}$, K. Fujita ${ }^{3}$, R. Fujiwara ${ }^{3}$, M. Fukushima ${ }^{6}$, R. Fukushima ${ }^{3}$, G. Furlich ${ }^{2}$, R. Gonzalez ${ }^{2}$, W. Hanlon ${ }^{2}$, M. Hayashi ${ }^{8}$, N. Hayashida ${ }^{9}$, K. Hibino ${ }^{9}$, R. Higuchi ${ }^{6}$, K. Honda ${ }^{10}$, D. Ikeda ${ }^{9}$, T. Inadomi ${ }^{11}$, N. Inoue ${ }^{12}$, T. Ishii ${ }^{10}$, H. Ito $^{13}$, D. Ivanov ${ }^{2}$, H. Iwakura ${ }^{11}$, A. Iwasaki ${ }^{3}$, H.M. Jeong ${ }^{14}$, S. Jeong ${ }^{14}$, C.C.H. Jui ${ }^{2}$, K. Kadota ${ }^{15}$, F. Kakimoto ${ }^{9}$, O. Kalashev ${ }^{16}$, K. Kasahara ${ }^{17}$, S. Kasami ${ }^{18}$, H. Kawai ${ }^{19}$, S. Kawakami ${ }^{3}$, S. Kawana ${ }^{12}$, K. Kawata ${ }^{6}$, I. Kharuk ${ }^{16}$, E. Kido ${ }^{13}$,

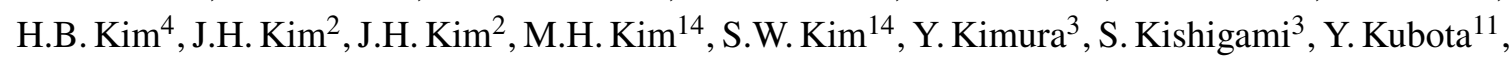
S. Kurisu ${ }^{11}$, V. Kuzmin ${ }^{16}$, M. Kuznetsov ${ }^{16,20}$, Y.J. Kwon ${ }^{21}$, K.H. Lee ${ }^{14}$, B. Lubsandorzhiev ${ }^{16}$, J.P. Lundquist ${ }^{2,22}$, K. Machida ${ }^{10}$, H. Matsumiya ${ }^{3}$, T. Matsuyama ${ }^{3}$, J.N. Matthews ${ }^{2}$, R. Mayta ${ }^{3}$, M. Minamino ${ }^{3}$, K. Mukai ${ }^{10}$, I. Myers ${ }^{2}$, S. Nagataki ${ }^{13}$, K. Nakai $^{3}$, R. Nakamura ${ }^{11}$, T. Nakamura ${ }^{23}$, T. Nakamura ${ }^{11}$, Y. Nakamura ${ }^{11}$, A. Nakazawa ${ }^{11}$, E. Nishio ${ }^{18}$, T. Nonaka ${ }^{6}, \mathrm{H}_{\text {. Oda }}{ }^{3}, \mathrm{~S}_{\text {Ogio }}{ }^{3,24}$, M. Ohnishi ${ }^{6}$, H. Ohoka ${ }^{6}$, Y. Oku ${ }^{18}$, T. Okuda ${ }^{25}$, Y. Omura ${ }^{3}$, M. Ono ${ }^{13}$, R. Onogi ${ }^{3}$, A. Oshima ${ }^{3}$, S. Ozawa ${ }^{26}$, I.H. Park ${ }^{14}$, M. Potts ${ }^{2}$, M.S. Pshirkov ${ }^{16,27}$, J. Remington ${ }^{2}$, D.C. Rodriguez ${ }^{2}$, G.I. Rubtsov ${ }^{16}$, D. Ryu ${ }^{28}$, H. Sagawa ${ }^{6}$, R. Sahara ${ }^{3}$, Y. Saito ${ }^{11}$, N. Sakaki ${ }^{6}$, T. Sako ${ }^{6}$, N. Sakurai ${ }^{3}$, K. Sano ${ }^{11}$, K. Sato ${ }^{3}$, T. Seki ${ }^{11}$, K. Sekino ${ }^{6}$, P.D. Shah ${ }^{2}$, Y. Shibasaki ${ }^{11}$, F. Shibata ${ }^{10}$, N. Shibata ${ }^{18}$, T. Shibata ${ }^{6}$, H. Shimodaira ${ }^{6}$, B.K. Shin ${ }^{28}$, H.S. Shin ${ }^{6}$, D. Shinto ${ }^{18}$, J.D. Smith ${ }^{2}$, P. Sokolsky ${ }^{2}$, N. Sone ${ }^{11}$,

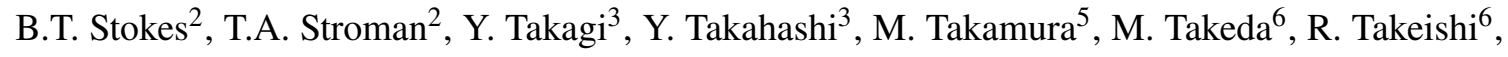
A. Taketa ${ }^{29}$, M. Takita ${ }^{6}$, Y. Tameda ${ }^{18}$, H. Tanaka ${ }^{3}$, K. Tanaka ${ }^{30}$, M. Tanaka ${ }^{31}$, Y. Tanoue ${ }^{3}$,

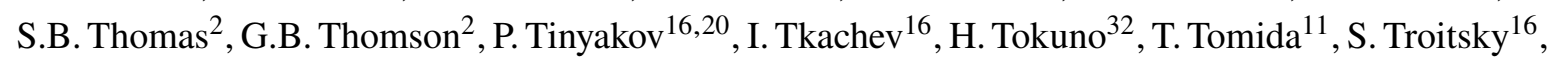

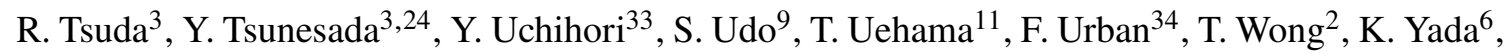
M. Yamamoto ${ }^{11}$, K. Yamazaki ${ }^{9}$, J. Yang ${ }^{35}$, K. Yashiro ${ }^{5}$, F. Yoshida ${ }^{18}$, Y. Yoshioka ${ }^{11}$, Y. Zhezher ${ }^{6,16}$, and Z. Zundel ${ }^{2}$

${ }^{1}$ Department of Physics, Loyola University Chicago, Chicago, Illinois, USA

${ }^{2}$ High Energy Astrophysics Institute and Department of Physics and Astronomy, University of Utah, Salt Lake City, Utah, USA

${ }^{3}$ Graduate School of Science, Osaka City University, Osaka, Osaka, Japan

${ }^{4}$ Department of Physics and The Research Institute of Natural Science, Hanyang University, Seongdong-gu, Seoul, Korea

${ }^{5}$ Department of Physics, Tokyo University of Science, Noda, Chiba, Japan

${ }^{6}$ Institute for Cosmic Ray Research, University of Tokyo, Kashiwa, Chiba, Japan

7 The Hakubi Center for Advanced Research and Graduate School of Science, Kyoto University, Kitashirakawa-Oiwakecho, Sakyo-ku, Kyoto, Japan

${ }^{8}$ Information Engineering Graduate School of Science and Technology, Shinshu University, Nagano, Nagano, Japan

${ }^{9}$ Faculty of Engineering, Kanagawa University, Yokohama, Kanagawa, Japan

${ }^{10}$ Interdisciplinary Graduate School of Medicine and Engineering, University of Yamanashi, Kofu, Yamanashi, Japan

${ }^{11}$ Academic Assembly School of Science and Technology Institute of Engineering, Shinshu University, Nagano, Nagano, Japan 
12 The Graduate School of Science and Engineering, Saitama University, Saitama, Saitama, Japan

${ }^{13}$ Astrophysical Big Bang Laboratory, RIKEN, Wako, Saitama, Japan

${ }^{14}$ Department of Physics, SungKyunKwan University, Jang-an-gu, Suwon, Korea

${ }^{15}$ Department of Physics, Tokyo City University, Setagaya-ku, Tokyo, Japan

${ }^{16}$ Institute for Nuclear Research of the Russian Academy of Sciences, Moscow, Russia

${ }^{17}$ Faculty of Systems Engineering and Science, Shibaura Institute of Technology, Minato-ku, Tokyo, Japan

18 Department of Engineering Science, Faculty of Engineering, Osaka Electro-Communication University, Neyagawa-shi, Osaka, Japan

${ }^{19}$ Department of Physics, Chiba University, Chiba, Chiba, Japan

${ }^{20}$ Service de Physique Théorique, Université Libre de Bruxelles, Brussels, Belgium

${ }^{21}$ Department of Physics, Yonsei University, Seodaemun-gu, Seoul, Korea

${ }^{22}$ Center for Astrophysics and Cosmology, University of Nova Gorica, Nova Gorica, Slovenia

${ }^{23}$ Faculty of Science, Kochi University, Kochi, Kochi, Japan

${ }^{24}$ Nambu Yoichiro Institute of Theoretical and Experimental Physics, Osaka City University, Osaka, Osaka, Japan

${ }^{25}$ Department of Physical Sciences, Ritsumeikan University, Kusatsu, Shiga, Japan

${ }^{26}$ Quantum ICT Advanced Development Center, National Institute for Information and Communications Technology, Koganei, Tokyo, Japan

${ }^{27}$ Sternberg Astronomical Institute, Moscow M.V. Lomonosov State University, Moscow, Russia

${ }^{28}$ Department of Physics, School of Natural Sciences, Ulsan National Institute of Science and Technology, UNIST-gil, Ulsan, Korea

${ }^{29}$ Earthquake Research Institute, University of Tokyo, Bunkyo-ku, Tokyo, Japan

${ }^{30}$ Graduate School of Information Sciences, Hiroshima City University, Hiroshima, Hiroshima, Japan

${ }^{31}$ Institute of Particle and Nuclear Studies, KEK, Tsukuba, Ibaraki, Japan

32 Graduate School of Science and Engineering, Tokyo Institute of Technology, Meguro, Tokyo, Japan

${ }^{33}$ Department of Research Planning and Promotion, Quantum Medical Science Directorate, National Institutes for Quantum and Radiological Science and Technology, Chiba, Chiba, Japan

${ }^{34}$ CEICO, Institute of Physics, Czech Academy of Sciences, Prague, Czech Republic

${ }^{35}$ Department of Physics and Institute for the Early Universe, Ewha Womans University, Seodaaemungu, Seoul, Korea 


\section{Acknowledgements}

The Telescope Array experiment is supported by the Japan Society for the Promotion of Science(JSPS) through Grants-in-Aid for Priority Area 431, for Specially Promoted Research JP21000002, for Scientific Research (S) JP19104006, for Specially Promoted Research JP15H05693, for Scientific Research (S) JP15H05741 and JP19H05607, for Science Research (A) JP18H03705, for Young Scientists (A) JPH26707011, and for Fostering Joint International Research (B) JP19KK0074, by the joint research program of the Institute for Cosmic Ray Research (ICRR), The University of Tokyo; by the Pioneering Program of RIKEN for the Evolution of Matter in the Universe (r-EMU); by the U.S. National Science Foundation awards PHY-1404495, PHY-1404502, PHY-1607727, PHY-1712517, PHY-1806797 and PHY-2012934; by the National Research Foundation of Korea (2017K1A4A3015188, 2020R1A2C1008230, \& 2020R1A2C2102800) ; by the Ministry of Science and Higher Education of the Russian Federation under the contract 075-15-2020-778, RFBR grant 20-02-00625a (INR), IISN project No. 4.4501.18, and Belgian Science Policy under IUAP VII/37 (ULB). This work was partially supported by the grants ofThe joint research program of the Institute for Space-Earth Environmental Research, Nagoya University and Inter-University Research Program of the Institute for Cosmic Ray Research of University of Tokyo. The foundations of Dr. Ezekiel R. and Edna Wattis Dumke, Willard L. Eccles, and George S. and Dolores Doré Eccles all helped with generous donations. The State of Utah supported the project through its Economic Development Board, and the University of Utah through the Office of the Vice President for Research. The experimental site became available through the cooperation of the Utah School and Institutional Trust Lands Administration (SITLA), U.S. Bureau of Land Management (BLM), and the U.S. Air Force. We appreciate the assistance of the State of Utah and Fillmore offices of the BLM in crafting the Plan of Development for the site. Patrick A. Shea assisted the collaboration with valuable advice and supported the collaboration efforts. The people and the officials of Millard County, Utah have been a source of steadfast and warm support for our work which we greatly appreciate. We are indebted to the Millard County Road Department for their efforts to maintain and clear the roads which get us to our sites. We gratefully acknowledge the contribution from the technical staffs of our home institutions. An allocation of computer time from the Center for High Performance Computing at the University of Utah is gratefully acknowledged. 